\title{
Retinal nerve fibre layer thickness - a biomarker of early dementia?
}

\section{6 \\ retinal \\ abnormalities \\ ... begin to \\ manifest in the \\ early stages \\ of cognitive \\ decline}
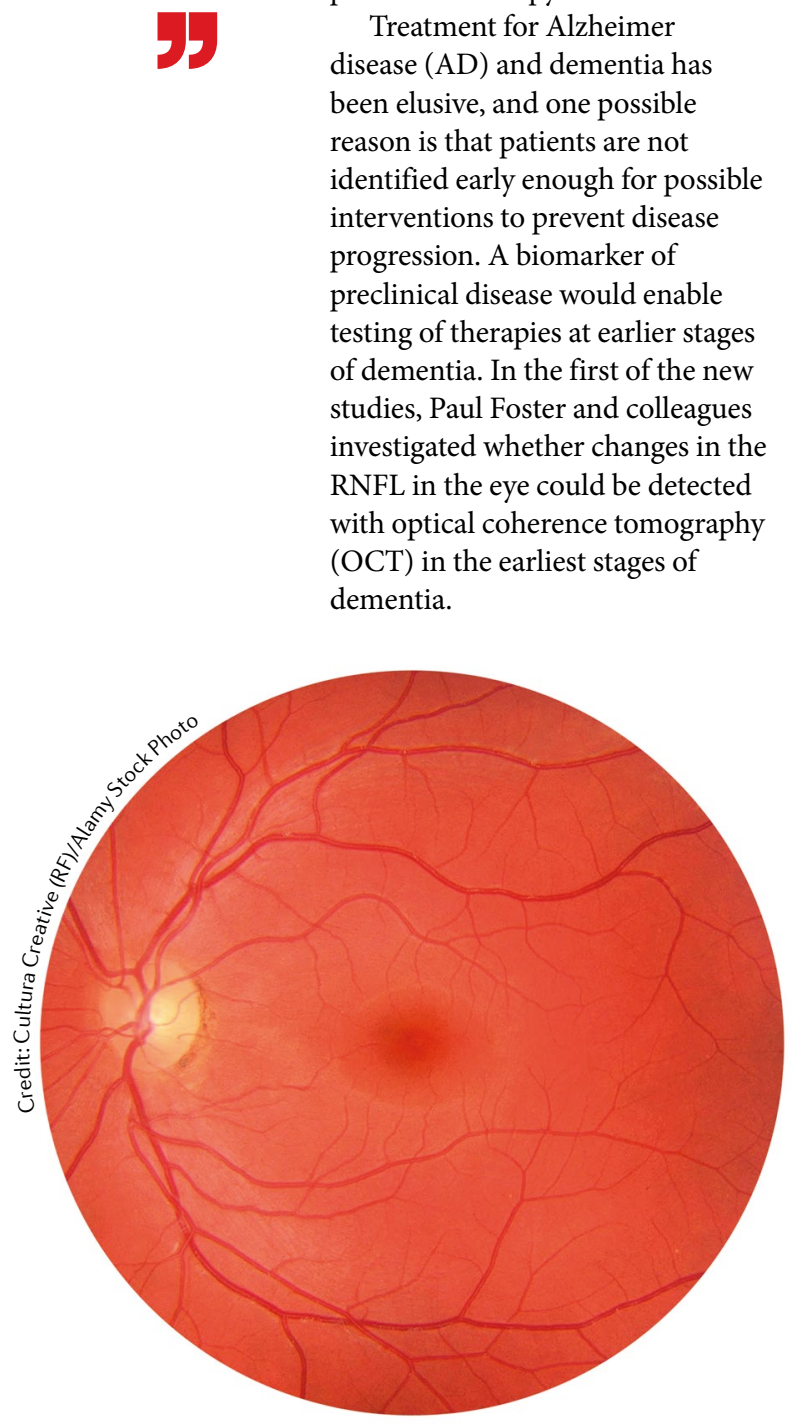

A thin retinal nerve fibre layer

(RNFL) is associated with an

increased risk of developing

dementia, according to two new

studies published together in JAMA

Neurology. The findings suggest

that retinal neurodegeneration

could be a biomarker of preclinical

dementia that could identify patients

who would benefit from early,

preventive therapy.

Treatment for Alzheimer disease $(\mathrm{AD})$ and dementia has been elusive, and one possible reason is that patients are not identified early enough for possible interventions to prevent disease progression. A biomarker of preclinical disease would enable testing of therapies at earlier stages of dementia. In the first of the new studies, Paul Foster and colleagues investigated whether changes in the RNFL in the eye could be detected with optical coherence tomography (OCT) in the earliest stages of dementia.
"It is well known that there are degenerative changes in the retina and optic nerve in dementia," explains Foster. "Our primary motivation was to determine if the RNFL-cognition relationship held true in the earliest stages of cognitive decline."

The study included 32,038 participants in the UK Biobank who had a mean age of 56 years at baseline. The researchers analysed OCT measurements and cognitive test scores collected at the baseline examination. A thinner RNFL was associated with poorer cognitive function: people whose RNFL thickness was in the lowest two quintiles were approximately twice as likely as those in the top quintile to have mild cognitive impairment.

For 1,251 patients, repeat assessments were performed at 3 years after baseline, and these follow-up data were compared with baseline data to determine whether RNFL thickness can predict future cognitive function.

"We found that those with a thinner RNFL were twice as likely to suffer cognitive decline over the next 3 years," says Foster. "Our findings suggest that the retinal abnormalities that are identifiable in established dementia begin to manifest in the early stages of cognitive decline."

These findings are reflected in the second study, led by Kamran Ikram, in which two OCT measurements were analysed to determine their association with prevalent and incident dementia. A total of 3,289 individuals aged $\geq 45$ years were included in the analysis, 41 of whom already had dementia. Baseline measurements of RNFL thickness and ganglion cell-inner plexiform layer (GC-IPL) thickness were taken between 2007 and 2012, and participants were followed up until 2015.

A thinner GC-IPL was associated with prevalent dementia, replicating previous findings. No association was found between RNFL thickness and prevalent dementia, but a thinner RNFL at baseline did indicate an increased risk of incident dementia and AD over the follow-up period.

"In patients with dementia, damage to brain regions covering the visual tract may cause retrograde degeneration of the optic nerve," the investigators write in their paper. “... this neurodegenerative process may manifest itself in the retina initially as thinner RNFL, after which thinning of the GC-IPL follows."

Although retinal degeneration has previously been observed in patients with $\mathrm{AD}$ and dementia, these two studies are the first to show that thinning of the RNFL can predict future cognitive decline. Together, the findings suggest that measures of retinal degeneration could serve as biomarkers of preclinical dementia, which Foster says could have important clinical benefits.

"We believe OCT measures of the retina will help identify those at highest risk of very early cognitive changes," Foster says. "This will, in turn, help to develop better clinical trials, and pull through new treatments more rapidly to patients."

Ian Fyfe

ORIGINAL ARTICLES Fang, K. et al. Association of retinal nerve fiber layer thinning with current and future cognitive decline. A study using optical coherence tomography. JAMA Neurol. https:// doi.org/10.1001/jamaneurol.2018.1578 (2018)| Mutlu, U. et al. Association of retinal neurodegeneration on optical coherence tomography with dementia. A population-based study.JAMA Neurol. https://doi.org/10.1001/ jamaneurol.2018.1563 (2018) 